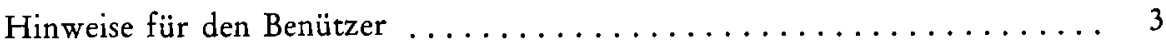



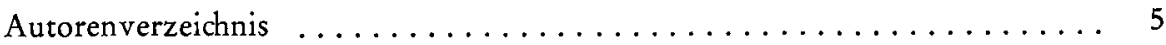

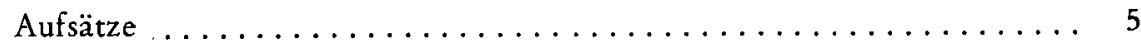

Kritiken, Berichte $\ldots \ldots \ldots \ldots \ldots \ldots \ldots \ldots \ldots \ldots \ldots \ldots$

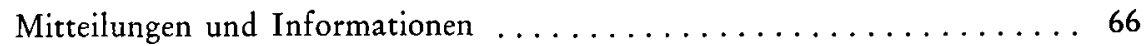

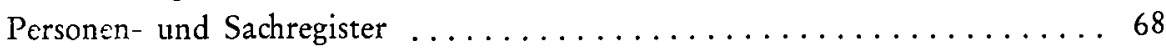

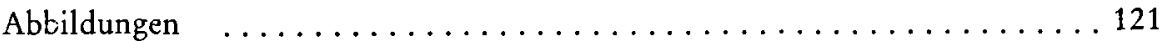



Aufsätze ............................. 147

Rezensionen $\ldots \ldots \ldots \ldots \ldots \ldots \ldots \ldots \ldots \ldots \ldots \ldots \ldots \ldots$

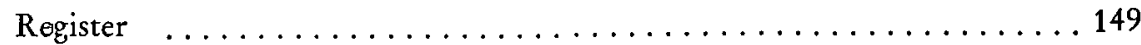

\title{
HINWEISE FÜR DEN BENÜTZER
}

Der vorliegende Registerband erschließt das Material von 25 Jahren wissenschaftlicher und kommentierender Tätigkeit der Osterreichischen Musikzeitschrift. Das Autorenverzeichnis ist unter Bedachtnahme auf Ubersichtlichkeit in "Aufsätze“, „Kritiken und Berichte" sowie meist anonyme „Mitteilungen und Informationen" gegliedert. Die Reihung erfolgt in den beiden ersten Teilen alphabetisch nach Autoren, wobei mehrere Beiträge desselben Verfassers chronologisch aufeinanderfolgen. Nur im Abschnitt „Mitteilungen und Informationen“ herrscht ausschließliche Chronologie. Um Rückverweise vom Personen- und Sachregister zu ermöglichen, ist jeder Artikel mit einem Sigel, bestehend aus dem Anfangsbuchstaben des Autors - im ersten Teil groß, im zweiten klein - und einer Zahl versehen, im dritten Teil nur mit Kleinbuchstabensigel (Abkürzungen der einzelnen Rubriken).

In das Personen- und Sachregister wurden alle Beiträge, detailliert nach Personen und Schlagworten aufgeschlüsselt, alphabetisch aufgenommen. Das den Jahrgangsund Seitenzahlen vorangestellte Autoren- bzw. Mitteilungssigel ermöglicht eine sofortige Identifizierung mit dem dazugehörigen Artikel (Sigel, Jahrgang/Seite). Unter dem umfangreichen Schlagwort "Musikleben (aktuelle Berichte) “ wurden Opern nur bei ausführlicher Besprechung einzeln angeführt; summarische Rezensionen finden daher nur unter dem jeweiligen Aufführungsort Berücksichtigung.

Das Abbildungsverzeichnis ist ebenfalls als Personen- und Sachregister angelegt, wobei die Zahlen nach dem jeweiligen Stichwort wieder Jahrgang und Seite der betreffenden Illustration anzeigen.

Der Abschnitt Phono-Schallplatten ist nach gleichem Prinzip wie der Hauptteil gestaltet: einem Verzeichnis von Aufsätzen und Rezensionen folgt wieder ein Register, das analog dem Hauptregister ebenfalls eine gleichzeitige Orientierung über den Verfasser eines bestimmten Beitrages gestattet.

Wien, im Februar 1975

Christa Harten-Flamm 